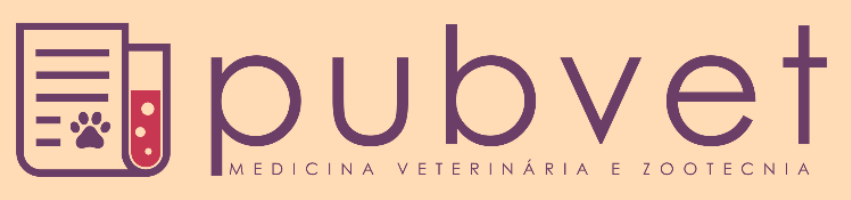

https://doi.org/10.31533/pubvet.v14n11a683.1-5

\title{
Avaliação do equilíbrio dos cascos de equinos criados a pasto e estabulados na Bahia
}

\author{
Fransael Franklyn Araújo da Silva ${ }^{1 *} \bullet$, Liliane Aparecida Oliveira de Paula ${ }^{1} \bullet$, Maristela de \\ Cássia Seudo Lopes $^{2} \bullet$ \\ ${ }^{1}$ Residentes do Setor de Clínica Médica e Cirúrgica de Grandes Animais do Hospital Veterinário da Universidade Federal do Paraná - Setor \\ Palotina, Palotina, Paraná, Brasil. \\ ${ }^{2}$ Docente do Departamento de Ciências Veterinárias da Universidade Federal do Paraná - Setor Palotina, Palotina, Paraná, Brasil \\ *Autor para correspondência, E-mail: fransael20@gmail.com
}

Resumo. Objetivou-se avaliar o equilíbrio dos cascos de equinos criados a pasto e estabulados no litoral norte da Bahia. Para isso 43 equinos, sem raça definida, peso médio de 300 a $400 \mathrm{~kg}$, ambos os sexos, sendo 21 animais criados a campo na Fazenda Campo Grande em Crisópolis e na Fazenda experimental da Universidade Federal da Bahia em Entre- Rios e 22 cavalos criados em estábulos na Cavalaria da Polícia Militar da Bahia. Foram avaliadas as seguintes mensurações: comprimento de pinça (p), altura de talão (t), comprimento (a) e largura de sola (b) e ângulo do casco (A) dos membros torácicos e pélvicos. Constatou-se que os animais estabulados apresentaram maior comprimento, menor largura de sola e menor altura de talão, porém sem diferença no comprimento de pinça e ângulo do casco de ambos os membros pélvicos e torácicos. Independentemente do manejo, constatou-se que os membros torácicos apresentam maiores valores nas medidas $\mathrm{p}, \mathrm{t}, \mathrm{a}$ e $\mathrm{b}$ e menores valores em $\mathrm{A}$, demonstrando serem maiores que os membros pélvicos. Embora o casqueamento e ferrageamento sejam empregados para manutenção do equilíbrio dos cascos, no presente estudo os animais mantidos em baia apresentaram cascos menos largos e mais compridos do que os criados a pasto, demonstrando que os desequilíbrios das medições do casco foram mais significativos nos animais estabulados, ou seja, nos animais que são casqueados frequentemente, o que pode acarretar em problemas futuros.

Palavras chave: balanceamento do casco, cavalo, podal

\section{Evaluation of the balance of equine hoof reared on grass and stacked in Bahia}

Abstract. The objective of this study was to evaluate the balance of the hooves of horses reared on pasture and sated on the northern coast of Bahia. For this, 43 horses, without defined breed, average weight of 300 to $400 \mathrm{~kg}$, both sexes, 21 animals reared in the field at Campo Grande Farm in Crisópolis and at the Experimental Farm of the Federal University of Bahia in Entre- Rios and 22 horses raised in stables in the Cavalary of the Military Police of Bahia. The following measurements were evaluated: tweezer length (p), bead height (t), length (a) and sole width (b) and angle of the hull (A) of the thoracic and pelvic limbs. It was found that the animals were tabulated with longer length, smaller sole width and lower bead height, but with no difference in tweezer length and hull angle of both pelvic and thoracic limbs. Regardless of management, it was found that the thoracic limbs present higher values in measures $\mathrm{p}, \mathrm{t}, \mathrm{a}$ and $\mathrm{b}$ and lower values in $\mathrm{A}$, demonstrating to be larger than the pelvic limbs. Although casing and ironing are used to maintain the balance of the hulls, in the present study, the animals kept in a stall presented smaller and longer hooves than those reared on pasture, demonstrating that imbalances in hoof 
measurements were more significant in the animals kept in stall, that is, in animals that are frequently casqueted, which can lead to future problems.

Keywords: hull balancing, horse, foot

\section{Evaluación del equilibrio de los cascos de caballos criados en pastos y estabulados en Bahía}

Resumen. El objetivo de este estudio fue evaluar el equilibrio de los cascos de los caballos criados en pastos y estabulados en la costa norte de Bahía. 43 caballos, sin raza definida, peso medio de 300 a $400 \mathrm{~kg}$, ambos sexos, 21 animales criados en el campo en la Granja Campo Grande en Crisópolis y en la Granja Experimental de la Universidad Federal de Bahía en Entre-Ríos y 22 caballos criados en establos en la Caballería de la Policía Militar de Bahía. Se evaluaron las siguientes medidas: longitud de la pinza (p), altura del cordón (t), longitud (a) y anchura de la suela (b) y ángulo del casco (A) de las extremidades torácicas y pélvicas. Se encontró que los animales estabulados prestaban mayor longitud, ancho de la suela más pequeña y menor altura del talón, pero sin diferencia en la longitud de la pinza y el ángulo del casco de las extremidades pélvicas y torácicas. Independientemente de la gestión, se encontró que las extremidades torácicas presentan valores más altos en las medidas $\mathrm{p}, \mathrm{t}$, a y b y valores más bajos en $\mathrm{A}$, demostrando ser más grande que las extremidades pélvicas. Aunque el cuidado del casco y herraje se utilizan para mantener el equilibrio de los cascos, en el presente estudio los animales mantenidos en establo tenían pezuña más pequeñas y largas que los criados en pastos, demostrando que los desequilibrios en las mediciones de los cascos eran más significativos en los animales criados en establos, es decir, en animales que son herrados frecuentemente lo que puede conducir a problemas futuros.

Palabras clave: equilibrio de pies, caballo, casco

\section{Introdução}

A locomoção dos equinos, semelhante a outras espécies, se faz a partir de mecanismos biomecânicos, resultantes do atrito dos cascos com o solo e deslocamento do centro de gravidade. A resultante da atuação das forças musculares incide sobre a região dos cascos colaborando para o desgaste e adaptação deles. Devido à domesticação da espécie e a característica do casco de crescimento contínuo, é comum que não ocorra o desgaste natural suficiente para a manutenção do equilíbrio dos cascos, podendo o mesmo ser substituído pelo casqueamento (Nicoletti et al., 2000).

O casqueamento e ferrageamento quando realizados de forma errônea, ou associados a problemas de conformação dos cascos, podem gerar desequilíbrios que comprometem a distribuição das forças e os mecanismos de absorção da concessão nos cascos, sendo causa de dor direta ou ainda uma causa de dor indireta em uma região proximal do membro (Snow \& Birdsall, 1990; T A Turner, 1993).

A avaliação do equilíbrio podal pode ser realizada a partir de medidas do casco, como altura de pinça, altura de talão, comprimento de casco, largura de casco, ângulo de casco, entre outras (Turner, 2003). Dentre as alterações do equilíbrio podal, revela-se a contração dos talões e ranilha, desnivelamento dorso-palmar e médio-lateral, tamanho do casco em relação ao peso do animal e diferença entre o ângulo da pinça dos cascos contralaterais (Redden, 1989; T A Turner \& Stork, 1989).

Sabe-se que o equilíbrio dos cascos é influenciado pelo manejo de produção, tipo de criação, alimentação, tipo de trabalho realizado pelo animal, raça, peso e tipo de solo (Melo et al., 2006; Stachurska et al., 2008).

Entretanto, não foram totalmente elucidadas as diferenças entre o equilíbrio de casco de equinos criados a pasto e/ou mantidos estabulados. Diante dessa situação e partindo-se da hipótese de que animais criados a pasto possuíssem cascos menos equilibrados devido à característica de um solo menos abrasivo e menos episódios de casqueamentos, objetivou-se descrever as medidas dos cascos de equinos criados em dois sistemas: a campo e estabulados, inferindo sob o equilíbrio dos mesmos. 


\section{Material e métodos}

Os procedimentos foram aprovados pela Comissão de Ética em Experimentação Animal da EMVZ - UFBA, Salvador, com o número 74/2016. Foram utilizados 43 equinos adultos (20 fêmeas e 23 machos) pertencentes à EMVZ- UFBA, Cavalaria da Polícia Militar da Bahia e Fazenda Campo Grande, sem raça definida, com média de 300 a $400 \mathrm{~kg}$. Os animais foram divididos em dois grupos, estabulados e criados a pasto. Os 22 animais estabulados (10 fêmeas e 12 machos) eram mantidos em baias individuais e alimentados com feno de capim coast cross, água ad libitum e ração de equinos para manutenção correspondente a $1 \%$ do peso vivo. Os 21 restantes (10 fêmeas e 11 machos) foram criados a pasto com capim-transvala, (Digitaria decumbens) água ad libitum, utilizando manejo extensivo. Procedeu-se as medições dos cascos nos membros torácicos e pélvicos. Nos animais que estavam utilizando ferraduras, não foram descontados a espessura das mesmas. As medidas constaram de: ângulo do casco (A); comprimento da pinça (p) e talão (t) (Figura 1). Comprimento da sola do casco (a) e largura da sola do casco (b) (Figura 2). As medidas de A foram obtidas com auxílio de um podogoniômetro e as medidas de a, b, p e t foram obtidas com uma régua metálica (Figura 3 ).

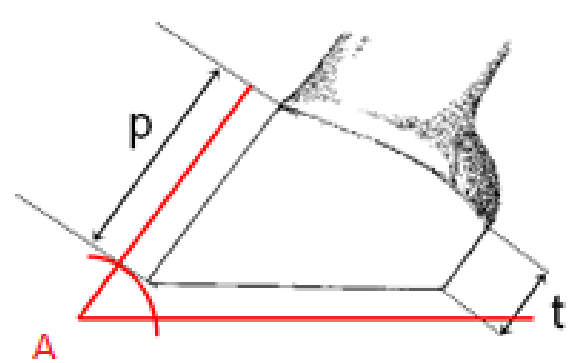

Figura 1. Esquema demonstrativo da vista lateral com as medidas obtidas dos cascos torácicos direito e esquerdo: ângulo do casco (A), comprimento da pinça (p) e comprimento do talão (t). Fonte: $\underline{\text { Hussni }}$ et al. $(\underline{2015})$.

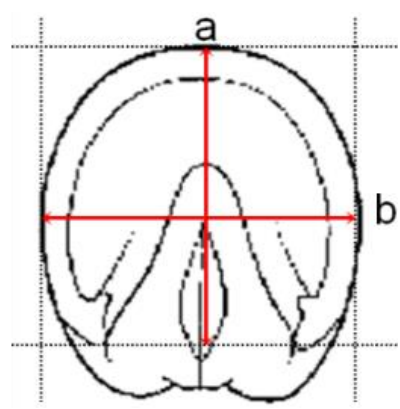

Figura 2. Esquema demonstrativo das medidas lineares compreendendo: comprimento do casco visto pela sola (a) e largura do casco visto pela sola (b).

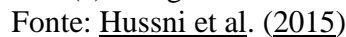

Os dados coletados foram tabulados e submetidos a análise estatística dos dados utilizando-se o software estatístico Sigma Plot ${ }^{\circledR}$ 11.0, onde foi realizado o "teste t" para variáveis com distribuição normal e o "teste Mann-Whitmey" para variáveis sem distribuição normal (teste não paramétrico). Selecionaram-se os animais em delineamento totalmente casualizado, adotando o nível de significância de $\mathrm{P}<0,05$.

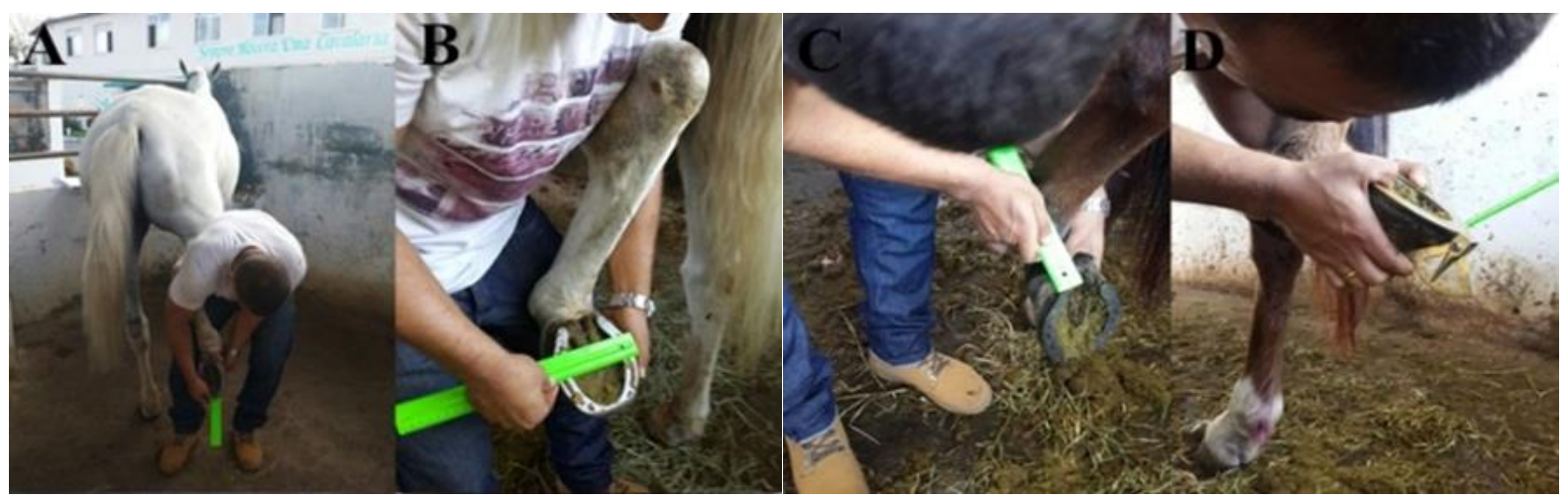

Figura 3. (A) Mensuração do comprimento de pinça (p), (B) largura de sola (b) e (C) altura de talão (c) utilizando uma régua, e (D) ângulo do casco (A) com auxílio de um podogoniômetro.

\section{Resultados e discussão}

Dentre as variantes analisadas, foram observadas diferenças entre os sistemas de criação (baia ou pasto), em largura de sola (b), comprimento de sola (a) e altura de talão (t). Contudo sem diferença entre comprimento de pinça (p) e ângulo de casco (A) em todos os membros avaliados (Tabela 1), ou seja, 
animais criados em baia, apresentaram cascos com maior comprimento, menor largura de sola e menor altura de talão.

Tabela 1. Valores médios $(\mathrm{X} \pm \mathrm{DP})$ ou medianos (max-min) das medidas de comprimento de pinça $(\mathrm{p})$, altura de talão $(\mathrm{t})$, comprimento (a) e largura (b) de sola em centímetros e ângulo do casco (A) nos membros torácicos e pélvicos (direito e esquerdo), de cavalos criados estabulados e a pasto.

\begin{tabular}{lcccccccc}
\hline \multirow{2}{*}{ Medidas } & \multicolumn{2}{c}{ MTE } & \multicolumn{2}{c}{ MTD } & \multicolumn{2}{c}{ MPE } & \multicolumn{2}{c}{ MPD } \\
& Pasto & Baia & Pasto & Baia & Pasto & Baia & Pasto & Baia \\
\hline $\mathrm{p}$ & $9,1 \pm 0,2^{\mathrm{A}}$ & $9,3 \pm 0,2^{\mathrm{A}}$ & $9,2 \pm 0,4^{\mathrm{A}}$ & $9,1 \pm 0,4^{\mathrm{A}}$ & $8,7(8-9)^{\mathrm{A}}$ & $8,5(8,0-8,9)^{\mathrm{A}}$ & $8,5(9-8)^{\mathrm{A}}$ & $8,5(8,9-8)^{\mathrm{A}}$ \\
$\mathrm{t}$ & $4(4,6-3,7)^{\mathrm{A}}$ & $3,8(4-3,7)^{\mathrm{B}}$ & $4(4,5-3,7)^{\mathrm{A}}$ & $4(4,2-3,5)^{\mathrm{B}}$ & $3,8(4-3,3)^{\mathrm{A}}$ & $3,3(3,6-3,1)^{\mathrm{B}}$ & $4(4-3,6)^{\mathrm{A}}$ & $3,0(3,5-3)^{\mathrm{B}}$ \\
$\mathrm{a}$ & $11,5 \pm 24^{\mathrm{A}}$ & $14 \pm 2,3^{\mathrm{B}}$ & $11,5 \pm 2^{\mathrm{A}}$ & $13 \pm 2^{\mathrm{B}}$ & $11 \pm 2,3^{\mathrm{A}}$ & $13 \pm 2,3^{\mathrm{B}}$ & $12 \pm 2,7^{\mathrm{A}}$ & $11 \pm 2,7^{\mathrm{B}}$ \\
$\mathrm{b}$ & $12,6 \pm 0,6^{\mathrm{A}}$ & $12 \pm 0,6^{\mathrm{B}}$ & $12,9 \pm 0,8^{\mathrm{A}}$ & $12,0 \pm 0,8^{\mathrm{B}}$ & $12(11,-12,9)^{\mathrm{A}}$ & $11(11,6-10,6)^{\mathrm{A}}$ & $11(11,5-10,6)^{\mathrm{A}}$ & $13(11,8-12,5)^{\mathrm{B}}$ \\
$\mathrm{A}$ & $49(48-49,6)^{\mathrm{A}}$ & $49(45-50)^{\mathrm{A}}$ & $49(50-46)^{\mathrm{A}}$ & $49(50-48)^{\mathrm{A}}$ & $50(50-49)^{\mathrm{A}}$ & $50(50,7-49)^{\mathrm{A}}$ & $50(50-49)^{\mathrm{A}}$ & $50(51-49)^{\mathrm{A}}$ \\
\hline
\end{tabular}

"Médias ou medianas seguidas por letras diferentes na mesma linha, diferem entre os grupos (pasto x estabulados) pelo "teste t" (dados paramétricos) e o "teste Mann-Whitmey" (dados não paramétricos).

De acordo com Stashak (1991) e Stashak (2011), o desgaste dos cascos sofre influência direta do tipo de solo que o animal vive, sendo assim a diferença nas variáveis (a) e (b) entre animais criados em baia ou pasto é justificada, pois os animais mantidos estabulados apresentavam-se de ferraduras, o que impossibilita o atrito do casco com o solo e evita seu desgaste natural.

Com relação à $(t)$, menores valores foram observados nos animais estabulados. Segundo Turner (2003), a razão entre (p) e (t) deve ser aproximadamente de 3:1 e de acordo com Stashak (1991) e Stashak (2011), quanto menor o (p), maior será o (t). Dessa forma, como os animais estabulados possuíam menores $(\mathrm{t})$, esperava-se que os mesmos apresentariam maiores $(\mathrm{p})$, fato esse não observado, sugerindo-se que esse fato provavelmente esteja correlacionado a um casqueamento inadequado.

Quando estipulada uma relação entre membros torácicos e pélvicos, observou-se diferença entre a maioria das variáveis estudadas (p, t, b, A) (Tabela 2).

Tabela 2. Valores médios $(\mathrm{X} \pm \mathrm{DP})$ ou medianos (max-min) das medidas de comprimento de pinça (p), altura de talão(t), comprimento (a) e largura (b) de sola em centímetros, e ângulo do casco (A) nos membros torácico direito (MTD), membro torácico esquerdo ( MTE), membro pélvico direito (MPD), membro pélvico esquerdo (MPE) de equinos criados a pasto e estabulados no litoral da Bahia.

\begin{tabular}{lcccc|cccc}
\hline Medidas & \multicolumn{4}{c|}{ Baia } & \multicolumn{4}{c}{ Pasto } \\
\cline { 2 - 9 } & MTD & MPD & MTE & MPE & MTD & MPD & MTE & MPE \\
\hline $\mathrm{p}$ & $9,1 \pm 0,7^{\mathrm{A}}$ & $8,4 \pm 0,4^{\mathrm{B}}$ & $9,3 \pm 0,4^{\mathrm{A}}$ & $8,4 \pm 0,4^{\mathrm{B}}$ & $9,2 \pm 0,9^{\mathrm{A}}$ & $8,4 \pm 0,6^{\mathrm{B}}$ & $9,1 \pm 0,8^{\mathrm{A}}$ & $8,6 \pm 0,7^{\mathrm{A}}$ \\
$\mathrm{t}$ & $3,9 \pm 0,6^{\mathrm{A}}$ & $3,2 \pm 0,4^{\mathrm{B}}$ & $3,8 \pm 0,4^{\mathrm{A}}$ & $3,4 \pm 0,3^{\mathrm{B}}$ & $4,0 \pm 3,7^{\mathrm{A}}$ & $4,0 \pm 3,6^{\mathrm{A}}$ & $4,2 \pm 0,8^{\mathrm{A}}$ & $3,9 \pm 0,9^{\mathrm{A}}$ \\
$\mathrm{a}$ & $13,6 \pm 0,9^{\mathrm{A}}$ & $13,6 \pm 0,7^{\mathrm{A}}$ & $13,9 \pm 0,8^{\mathrm{A}}$ & $13,4 \pm 0,7^{\mathrm{A}}$ & $12,9 \pm 0,8^{\mathrm{A}}$ & $12,1 \pm 0,8^{\mathrm{A}}$ & $12,6 \pm 0,8^{\mathrm{A}}$ & $12,1 \pm 1^{\mathrm{A}}$ \\
$\mathrm{b}$ & $11,9 \pm 0,8^{\mathrm{A}}$ & $11,1 \pm 0,6^{\mathrm{B}}$ & $12,0 \pm 0,6^{\mathrm{A}}$ & $11,1 \pm 0,6^{\mathrm{B}}$ & $11,6 \pm 0,8^{\mathrm{A}}$ & $10,9 \pm 0,6^{\mathrm{B}}$ & $11,5 \pm 0,9^{\mathrm{A}}$ & $11,0 \pm 0,6^{\mathrm{A}}$ \\
$\mathrm{A}$ & $49(50-46)^{\mathrm{A}}$ & $50(51-49)^{\mathrm{B}}$ & $49(50-45)^{\mathrm{A}}$ & $50(50-49)^{\mathrm{B}}$ & $49(50-48)^{\mathrm{A}}$ & $50(50-49)^{\mathrm{B}}$ & $49(50-48)^{\mathrm{A}}$ & $50(50-49)^{\mathrm{B}}$ \\
\hline
\end{tabular}

*Médias e medianas seguidas por letras diferentes na mesma linha, diferem entre os grupos (torácicos x pélvicos) dentro de cada grupo (Baia e pasto) pelo "teste t” (dados paramétricos) e o "teste Mann-Whitney” (dados não paramétricos).

Nas medidas de (A) (Tabela 1) não foram observadas diferenças entre os grupos estudados, pois não houve diferença entre as medidas (p). Contudo as medidas de (A) apresentam-se significativas (Tabela 2), sendo reflexa da significância nas medidas de (p). O (A) está diretamente ligado ao (p), no qual quanto maior o (p), menor será o (A). Vale-se ressaltar que os animais com (p) elevado prolongam o breakover e tensionam as estruturas de sustentação. Além disso, também podem contrair os talões, que mesmo não sendo objeto desse estudo são de extrema importância, pois formam uma concavidade na borda distal da parede do casco (Stashak, 1991; Stashak, 2011). Todavia, quando se compara os membros torácicos e pélvicos, independente do manejo adotado, observam-se valores que entram de acordo com o que Andrade (1986) relata, não pelos valores em si, mas sim por esses valores apresentarem-se sempre menores nos torácicos do que nos pélvicos.

As demais medidas significativas (Tabela 2) corroboram com Stashak (1991) e Stashak (2011), pois os cascos dos membros torácicos recebem a maior parte do peso de sustentação, sendo assim, tendem a 
ser mais circulares, enquanto os pélvicos, mais ovalados, ou seja, membros torácicos apresentaram maior (b) do que membros pélvicos, fato esse observado nesse estudo.

Contudo as medidas de (a) comparadas entre membros torácicos (MTs) e membros pélvicos (MPs) (Tabela 2) foram diferentes das encontradas em estudo realizado por Schade et al. (2013) onde avaliou biometria do equilíbrio podal em 44 animais e observou diferença significativa entre os mesmos. Essa ausência de significância nas medidas de (a), podem ser novamente justificadas pelo casqueamento inadequado dos animais estabulados e o não casqueamento regular dos animais não estabulados.

\section{Conclusão}

A partir dos resultados apresentados, observou-se que ao contrário do previsto, os animais criados em estábulos apresentaram os cascos menos balanceados do que os criados a pasto, mostrando que falhas no manejo, casqueamento ou ferrageamento inadequados ocasionam complicações podais. Diante disso, a falta do conhecimento teórico ainda é um fator preponderante para a ocorrência de altos índices dos desequilíbrios de casco. Assim, a mensuração de medidas objetivas do casco se mostra uma ferramenta útil e de fácil aplicação, que pode contribuir para diminuição da ocorrência de desequilíbrios podais.

\section{Referências bibliográficas}

Andrade, L. S. (1986). O condicionamento do equino no Brasil. In Recife: Equicenter (Vol. 201).

Hussni, C. A., Watanabe, M. J., Alonso, J., Rodrigues, C. A., Barbosa, L., \& Alves, A. L. G. (2015). Comparação morfológica dos cascos dos membros torácicos de equinos submetidos à tenotomia do flexor digital superficial ou à desmotomia de seu ligamento acessório. Veterinária Notícias, 21(2), 12-19. DOI: https://doi.org/10.14393/vtv21n2a2015.30898

Melo, U. P. de, Ferreira, C., Santiago, R. M. F. W., Palhares, M. S., \& Maranhão, R. P. A. (2006). Equilíbrio do casco equino. Ciência Animal Brasileira, 7(4), 389-398.

Nicoletti, J. L. M., Schlegel, C., Thomassian, A., Hussni, C. A., \& Alves, A. L. G. (2000). Mensuração do casco de equinos para identificação objetiva de anormalidades de conformação. Veterinária Notícias, 6(1), 61-68.

Redden, R. F. A. (1989). A method for treating clubfoob. Annual Convention of Theaaep, 321-324.

Schade, J., Baldissera, R., Paolini, E., \& Fonteque, J. H. (2013). Biometria do equilíbrio podal em equinos de tração pertencentes ao Programa de Extensão" Amigo do Carroceiro" do Centro de Ciências Agroveterinárias da Universidade do Estado de Santa Catarina no município de Lages/SC, Brasil. Ciência Rural, 43(3), 456-461. DOI: https://doi.org/10.1590/s0103-84782013000300013

Snow, V. E., \& Birdsall, D. P. (1990). Specific parameters used to evaluate hoof balance and support. Proceedings of the Annual Convention of the American Association of Equine Practitioners (USA), 299-311.

Stachurska, A., Kolstrung, R., Pieta, M., Silmanowicz, P., \& Klimorowska, A. (2008). Differentiation between fore and hind hoof dimensions in the horse (Equus caballus). Archives Animal Breeding, 51(6), 531-540. DOI: https://doi.org/10.5194/aab-51-531-2008

Stashak, T S. (1991). Equine wound management. Lea and febiger.

Stashak, Ted S. (2011). Claudicação em eqüinos segundo Adams. Editora Roca.

Turner, T A. (1993). The use of hoof measurements for the objective assessment of hoof balance. Proceedings of the Annual Convention of the American Association of Equine Practitioners (USA), 389-395.

Turner, T A, \& Stork, C. (1989). Hoof abnormalities and their relation to lameness. Proceedings of the Annual Convention of the American Association of Equine Practitioners (USA).

Turner, Tracy A. (2003). Examination of the equine foot. The Veterinary Clinics of North America. Equine Practice, 19(2), 309-332. DOI: https://doi.org/10.1016/s0749-0739(03)00023-3.

Recebido: 22 de maio, 2020

Aprovado: 25 de junho, 2020.

Disponível online: 23 de outubro, 2020.
Licenciamento: Este artigo é publicado na modalidade Acesso Aberto sob a licença Creative Commons Atribuição 4.0 (CC-BY 4.0), a qual permite uso irrestrito, distribuição, reprodução em qualquer meio, desde que o autor e a fonte sejam devidamente creditados. 